Article

\title{
Ensemble Methods for Jump-Diffusion Models of Power Prices
}

\author{
Carlo Mari ${ }^{1, *,+}+$ DD and Cristiano Baldassari ${ }^{2,+}$ \\ 1 Department of Economics, University of Chieti-Pescara, 65100 Pescara, Italy \\ 2 Department of Neuroscience, Imaging and Clinical Sciences, University of Chieti-Pescara, 66100 Chieti, Italy; \\ cristiano.baldassari@unich.it \\ * Correspondence: carlo.mari@unich.it; Tel.: +39-347-0826702 \\ + These authors contributed equally to this work.
}

check for

updates

Citation: Mari, C.; Baldassari, C. Ensemble Methods for Jump-Diffusion Models of Power Prices. Energies 2021, 14, 2084. https://doi.org/10.3390/en14082084

Academic Editor: Javier Contreras

Received: 4 March 2021

Accepted: 6 April 2021

Published: 9 April 2021

Publisher's Note: MDPI stays neutral with regard to jurisdictional claims in published maps and institutional affiliations.

Copyright: (c) 2021 by the authors. Licensee MDPI, Basel, Switzerland. This article is an open access article distributed under the terms and conditions of the Creative Commons Attribution (CC BY) license (https:// creativecommons.org/licenses/by/ $4.0 /)$.

\begin{abstract}
We propose a machine learning-based methodology which makes use of ensemble methods with the aims (i) of treating missing data in time series with irregular observation times and detecting anomalies in the observed time behavior; (ii) of defining suitable models of the system dynamics. We applied this methodology to US wholesale electricity price time series that are characterized by missing data, high and stochastic volatility, jumps and pronounced spikes. For missing data, we provide a repair approach based on the missForest algorithm, an imputation algorithm which is completely agnostic about the data distribution. To identify anomalies, i.e., turbulent movements of power prices in which jumps and spikes are observed, we took into account the no-gap reconstructed electricity price time series, and then we detected anomalous regions using the isolation forest algorithm, an anomaly detection method that isolates anomalies instead of profiling normal data points as in the most common techniques. After removing anomalies, the additional gaps will be newly filled by the missForest imputation algorithm. In this way, a complete and clean time series describing the stable dynamics of power prices can be obtained. The decoupling between the stable motion and the turbulent motion allows us to define suitable jump-diffusion models of power prices and to provide an estimation procedure that uses the full information contained in both the stable and the turbulent dynamics.
\end{abstract}

Keywords: power prices; spikes; jump-diffusion dynamics; mean-reversion; machine learning; missForest; isolation forest; anomaly detection

\section{Introduction}

Time series are occasionally observed at irregular observation times. Such irregular samples may occur naturally in climate research [1], in astronomy [2], in heart rate analysis [3] and even in financial time series [4]. The typical method applied to deal with irregular samples is to ignore them and literally close up the gaps. However, a missing values imputation (or gap filling) strategy can be informative and could provide fundamental knowledge for the subsequent stochastic analysis [5]. This is particularly true for financial time series. In such a case, although price time series are typically non stationary, log-return time series, computed as the difference in log-prices between two subsequent observations, have better behavior [6]. In the presence of missing data, log-returns computed over different time intervals may have different informative content. In fact, information affecting the dynamics of power prices can be released while the market is closed $[7,8]$. Moreover, we could encounter difficulties if we want to detect seasonality in market prices or compare markets with different closure day patterns.

In this paper, we will focus on the electricity prices of US power markets. US electricity price time series show irregular sampling (lack of daily data points) as a result of weekends, holidays and other missing data due to market specific reasons.

Existing methods for analyzing irregular time series can be categorized into three main directions [9]: (i) the repair approach in which missing observations are recovered via 
smoothing or imputation [10-14]—also implemented, especially in recent years, by machine learning methods [15-18]; (ii) the generalization of spectral analysis tools [19,20], such as wavelets [21-24]; (iii) kernel methods [25,26]. In this paper, we deal with a repair approach which uses an input preparation step based on machine learning. We work out this problem first by using a regular sampling grid layer over the original time series, and then by computing a value for each of new sampled point from the available samples, in order to have an equidistant missing-data problem [5]. For such an imputation process, we chose the missForest algorithm [27] that is completely agnostic about the data distribution. We verified that using this machine learning strategy for gap-filling, data quality did improve in a very efficient manner, especially compared to traditional methods. Therefore, being predominantly data-driven by design, we could rely just on training data and using very few parameters to reconstruct complete time series. Once the filling process of the observed power price time series is completed, the anomaly detection problem is addressed.

"An anomaly is an observation which deviates so much from the other observations as to arouse suspicions that it was generated by a different mechanism" [28]. Electricity price time series are prone to have anomalies: they can occur as a consequences of excess demand, power outages, communication failures, activation of circuit breakers at substations, meter malfunctions and other reasons [29,30]. The liberalization process of the electricity sector has significantly increased the price volatility [31]. Looking at the time series of electricity prices, we can see some very erratic behavior. Power prices show, in fact, variable and unpredictable behavior with high and stochastic volatility; jumps and pronounced spikes; and a strong mean-reversion component, responsible for reducing prices after a jump or a spike has occurred [32]. Specifically, electricity price time series are characterized by normal stable periods in which they fluctuate around a long-run mean and turbulent price movements in which the dynamics are affected by jumps and short-lived spikes of large magnitude. This complex dynamics produces non-normal empirical distributions of log-returns with high volatility values and non-zero skewness and high kurtosis values [33].

In this paper we provide a general methodology to detect the stable price dynamics and decouple them from the turbulent dynamics in which jumps and spikes, i.e., anomalous price movements, occur. Our starting point is to consider the reconstructed no-gap time series, filled by the missForest algorithm, as affected by anomalies that we are going to identify and remove. The anomaly identification process is carried out on the filled original time series of electricity prices by using the isolation forest (or iForest) algorithm [34], an anomaly detection method that isolates anomalies instead of profiling normal data points, as in the most common techniques [35]. Using this unsupervised method, we can detect abrupt changes or novelty in prices time series without using a "universal" definition, considering that we cannot provide a "standard" reference for anomaly in electricity prices time series. As for the lack of "good" (non-anomalous) benchmark time series, we prefer an agnostic approach. Moreover, since we want to reduce to the minimum the impact of parameter setting on the anomaly detection process, iForest is a particularly suitable algorithm for this purpose [36-38]. Once identified, anomalies can be removed from the dynamics. At the end of this process, the additional gaps created by removing the anomalous regions of the dynamics will be newly filled by the missForest imputation algorithm. In this way, we obtain: (i) a complete and clean time series describing the stable dynamics of power prices; (ii) a separation between the stable dynamics and the turbulent dynamics to feed the stochastic analysis with. This is the first contribution of the present paper to the literature.

Several models have been proposed in the literature to describe the dynamics of power prices observed in real markets. Since the seminal paper by Lucia and Schwartz [39], the literature on this topic has grown exponentially. Mean-reverting jump-diffusion processes have been proposed $[40,41]$ to account for the jumpy and spiky behavior of power prices. Regime-switching processes [42] have also been used with the aim of modeling the stable dynamics and the turbulent dynamics of power prices separately [43-45]. Compared to more complex regime-switching models, jump-diffusion models offer a good compromise 
between mathematical tractability and the physical description of the price dynamics. Their use can be considered as the simplest modeling methodology to describe non-Gaussian processes with stochastic volatility. However, the estimation procedure of jump-diffusion models on market data require some care in order to take into account in a proper way the various components of the dynamics [32]. When estimating a jump-diffusion model, the main difficulty is to determine which price variations are caused by jumps and which ones are caused by the diffusion component of the process. The easiest and most common way to deal with this problem is to fix a threshold according to which price variations are considered to be caused by jumps and spikes [44]. In this case, the threshold must be set according to some well defined (but arbitrary) criteria [46]. An alternative approach is to estimate the jump-diffusion model by maximum likelihood without filtering jumps first [40]. However, this technique allows one to reproduce the standard deviation of log-returns well but underestimates kurtosis [45]. The use of iForest algorithm is suitable for overcoming these difficulties. The decoupling of the price dynamics between the stable motion and the turbulent motion obtained by the machine learning techniques proposed in this paper, allows us to provide a suitable estimation procedure for both the diffusion component and the jump component of the model that makes use of the full information contained in both the stable and the turbulent dynamics. The estimation results show an interesting agreement with market data. This is the second contribution to the literature.

To our knowledge, this is the first study in which unsupervised machine learning techniques have been employed to detect jumps and spikes in power price time series, thereby allowing the possibility of accurately describing the observed dynamics using the jump-diffusion models. The workflow of the whole methodology is depicted in Figure 1.

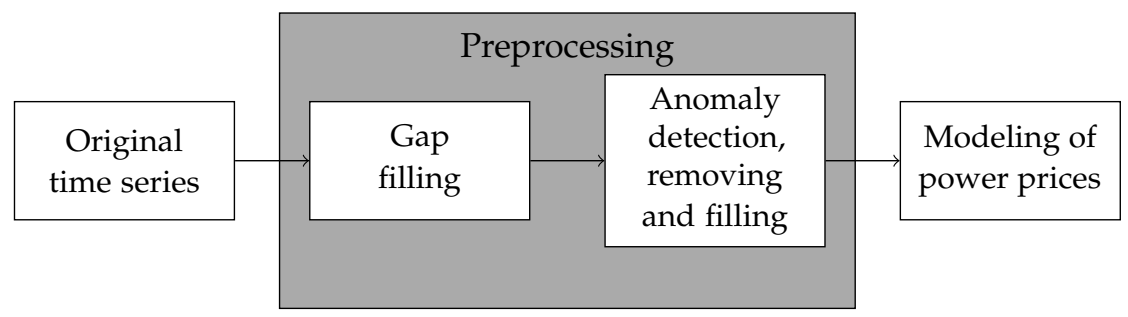

Figure 1. A block diagram of the whole methodology's workflow. Time series passing through a preprocessing block containing the gap filling (Section 2.1), anomaly detection, removing and filling (Section 2.2) phases. Thereafter, the following modeling of power prices (Section 3) was fed with the preprocessed time series.

The proposed approach has several advantages and potential applications. Specifically, our methodology offers the possibility to improve the data quality by using a data-driven approach, i.e., an unsupervised technique having as few parameters as possible, for the imputation of missing data and for the detections of anomalies in the dynamics [47-49]. Moreover, accurately modeling electricity price dynamics using simple models which can be easily calibrated on high quality data is essential for all the power market players [31]. The jump-diffusion model proposed in this paper is a short-term model and the short-term modeling of electricity prices is a central topic for both traders and producers in their attempts to hedge financial risk due to the unpredictability of power prices [50] by using power derivatives as well [51]. In this regard, having good short-term models of electricity prices capturing the first four central moments of log-return empirical distributions is of crucial importance for pricing power derivatives [52]. Moreover, modeling power prices over longer time horizons, ranging from a few years to decades, is strategically important for energy companies, in their efforts toward evaluating investments in capacity expansion and generating new technologies, and for policy makers involved in the energy planning decision making processes. In this regard, the proposed methodology can be employed as a long-term forecasting approach that allows us to derive the long-run behavior of power prices from their short-term dynamics. In the presence of a mean-reverting component, in fact, the probability distributions of power prices tend toward stationary long-run 
probability distributions [53]. In this way, the proposed approach also develops a robust link between the short-term and the long-term behavior of electricity prices.

The paper is organized as follows. Section 2 discusses the data processing methodology. Section 3 illustrates in some detail the mean-reverting jump-diffusion model used to describe the dynamics of power prices and the estimation procedure. Section 4 concludes. A comparison between the use of the missForest algorithm for gap filling purposes and a more traditional approach based on moving average techniques is provided in Appendix A.

\section{The Data Processing Methodology}

This section is devoted to discussing the data processing methodology. It is composed by two subsections in which we illustrate in detail all the steps of our procedure, namely, the filling process of the original wholesale time series of daily electricity prices, the anomaly detection process and the reconstruction of the stable motion time series.

\subsection{Gap Filling}

In our analysis we will consider daily electricity prices computed as weighted averages of the 24 hourly market prices. They are expressed in nominal dollars per megawatthour $(\$ / \mathrm{MWh})$. Let us, therefore, denote by $p(t)$ the daily price at time $t$ of $1 \mathrm{MWh}$ of electricity and by $M$ the number of observations of the original power price time series, one observation for each business day in which market data are available. We introduce, therefore, the following sets:

$$
\begin{array}{r}
p_{o b s}=\left\{p\left(\bar{t}_{1}\right), p\left(\bar{t}_{2}\right), \cdots, p\left(\bar{t}_{M}\right)\right\} \\
\mathbb{T}_{\text {obs }}=\left\{\bar{t}_{1}, \bar{t}_{2}, \cdots, \bar{t}_{M}\right\}
\end{array}
$$

where the set $p_{o b s}$ contains the original power price time series and $\mathbb{T}_{o b s}$ is the original time grid composed by $M$ daily positions. Then, we use a complete daily grid that includes, in addition to business days, weekends, holidays and all the other days with missing market data-hereinafter, market-closure days. In this way we expand the cardinality of both sets from $M$ to $N=M+C$, by introducing $C$ additional elements corresponding to the number of market-closure days. We set, therefore,

$$
\begin{array}{r}
p=\left\{p\left(t_{1}\right), p\left(t_{2}\right), \cdots, p\left(t_{N}\right)\right\} \\
\mathbb{T}=\left\{t_{1}, t_{2}, \cdots, t_{N}\right\}
\end{array}
$$

$\mathbb{T}$ being the complete set of daily ordered time grid positions. This operation produces a set of missing observations in correspondence with market-closure days. Hence we can split the data points into two subsets, namely, the observation set, $p_{o b s}$, and a set of missing values, $p_{m i s}$, given by the set difference,

$$
p_{\text {mis }}=p \backslash p_{\text {obs }},
$$

defined on the time set difference

$$
\mathbb{T}_{\text {mis }}=\mathbb{T} \backslash \mathbb{T}_{\text {obs }} .
$$

Now, to address the missing data problem in order to fill the gaps represented by the subset $p_{\text {mis }}$, we adopted an iterative imputation scheme based on the random forest technique [54]. In particular, we used the missForest algorithm [27] to fit a random forest model by taking the time series values belonging to $p_{o b s}$ as the outcome variables and the time values belonging to the set $\mathbb{T}_{o b s}$ as the input variables. After the fit, the missing values were imputed using prediction values from the fitted random forest algorithm, thereby determining the whole set $p$, i.e., the filled time series. 
Our dataset consists of daily time series of electricity prices observed in four US power markets in the period 1 January 2001 to 24 March 2020. Two power markets, namely, SP15 and Palo Verde (PV), are located in the US Southwest region: respectively, in Southern California (SP15) and in the Southwest (PV). The remaining two, namely, PJM and Nepool (NE) power markets, are located in the US Northeast region. Data are freely downloadable at www.eia.gov / electricity / wholesale (accessed on 27 March 2020). Figure 2 shows, as an example, the original time series (in blue) and the parts filled (in red) by the missForest algorithm on a complete daily grid for the 4-month period of September 2014 to December 2014.

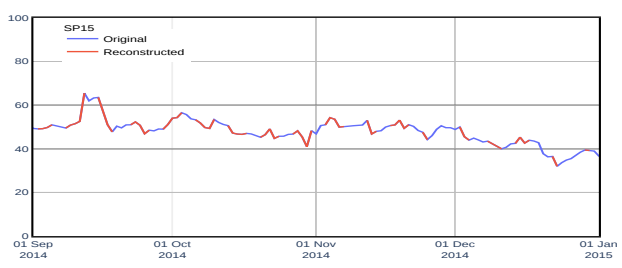

(a)

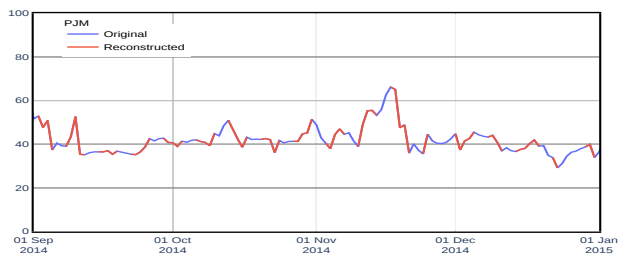

(c)

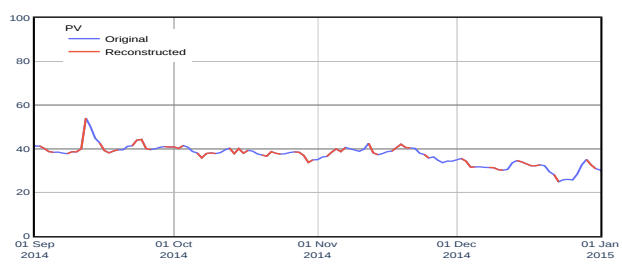

(b)

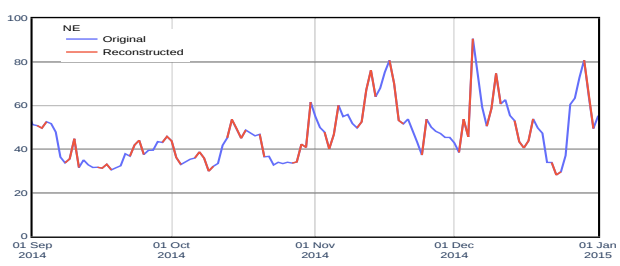

(d)

Figure 2. The original time series (in blue) and the parts filled (in red) by the missForest algorithm on a complete daily grid. The figure shows the 4-month period of September 2014 to December 2014. $x$-axis: calendar time; $y$-axis: electricity price. (a) SP15; (b) Palo Verde; (c) PJM; (d) Nepool.

Table 1 reports the number of days with missing data computed over the whole period under investigation, i.e., the period of 1 January 2001 to 24 March 2020. Table 2 depicts the values of the mean and standard deviation of the original and the filled time series' empirical distribution.

Table 1. Count of daily grid positions being empty (missing) or not (populated) in the period 1 January 2001 to 24 March 2020. Total grid positions: 7023.

\begin{tabular}{ccc}
\hline & Populated & Missing \\
\hline SP15 & 4638 & 2385 \\
PV & 4662 & 2361 \\
PJM & 4881 & 2142 \\
NE & 4493 & 2530 \\
\hline
\end{tabular}

Table 2. Mean and standard deviation (Std) values of the original and the filled time series empirical distribution.

\begin{tabular}{ccccc}
\hline & Original Mean & Filled Mean & Original Std & Filled Std \\
\hline SP15 & 50.83 & 51.94 & 34.13 & 37.09 \\
PV & 44.01 & 46.57 & 28.82 & 35.26 \\
PJM & 50.06 & 50.05 & 27.09 & 26.49 \\
NE & 56.52 & 56.85 & 32.19 & 31.52 \\
\hline
\end{tabular}


A comparison between the original time series empirical distribution and the filled time series empirical distribution is depicted in Figure 3.

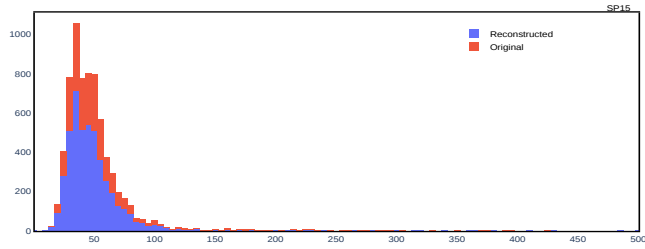

(a)

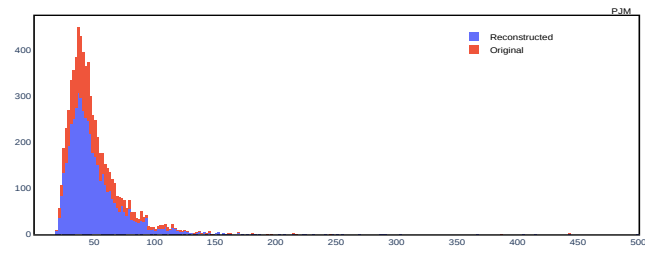

(c)

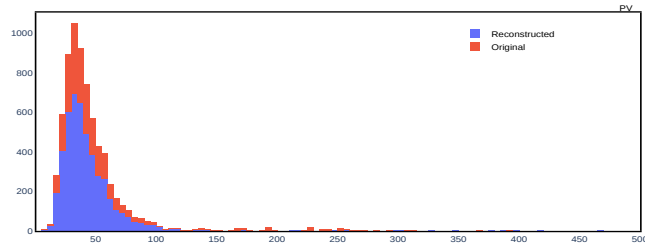

(b)

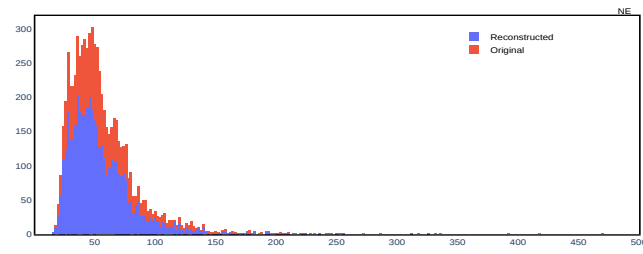

(d)

Figure 3. The original time series empirical distribution (in blue) and the filled time series distribution (in red). (a) SP15; (b) Palo Verde; (c) PJM; (d) Nepool.

We remark that this approach needs no tuning parameters, and hence it needs neither prior knowledge about the data nor assumptions about the distribution of the data of the variable domain [27]. Misztal [55] underlined the good performance of the missForest algorithm over generic missing patterns, including the case of Not missing at random (NMAR) data. According to Little and Rubin [56], there are three missing data mechanisms: missing completely at random (MCAR), missing at random (MAR) and not missing at random (NMAR). MCAR means that the probability of a piece of information being missing does not depend on $p_{m i s}$ or $p_{o b s}$; MAR means that the probability of a piece of information being missing does not depend on $p_{\text {mis }}$, but may depend on $p_{o b s}$; MNAR means that the probability of a piece of information being missing does depend on $p_{\text {mis }}$. Obviously, there is a risk of having artificial and compromised numerical effects which are inherent to any imputation method and that may result in further spurious effects [57]. However, missForest outperforms many methods of imputation, especially if data are supposed to describe complex interactions in which non-linear relations are suspected [27]. Empirical and simulation studies confirm that missForest method perform well and can produce unbiased parameter estimates and standard errors [58-61].

\subsection{Anomaly Detection, Removing and Filling}

Before addressing the anomaly detection problem, we performed a decomposition of the filled time series in order to extract the stochastic component of the power price dynamics. To do this, let us pose:

$$
s(t)=\ln p(t),
$$

the natural logarithm of the electricity price at time $t$. We assume that $s(t)$ is a linear superposition of a deterministic component, $f(t)$, accounting for trend and seasonality, and a random component, $x(t)$, namely,

$$
s(t)=f(t)+x(t) .
$$

Typically, electricity prices are higher in winter time and lower in summer time, so the seasonal component must account for this semiannual periodicity. A trend must be 
taken into account for expected inflation and conceivably for a real escalation rate of power prices (positive or negative). We used the STL decomposition technique [62] to identify the deterministic component of the dynamics, $f(t)$. STL stands for "seasonal and trend decomposition using LOESS" and separates the time series into a trend, a seasonal and a residual, stochastic component. LOESS stands for "locally estimated scatterplot smoothing" and it is a seasonal-trend decomposition procedure. Many excellent and comprehensive presentations of the STL decomposition technique can be found in the literature (see, e.g., ref. [63] and references therein). Figure 4 depicts (from top to bottom) the deterministic components, respectively, trend and seasonality, and the residual stochastic component of the filled time series of daily electricity log-prices, obtained from the STL decomposition technique, in the case of SP15 and Palo Verde power markets for the period 1 January 2001 to 24 March 2020. In Figure 5 the time series STL decomposition is shown in the case of PJM and Nepool power log-prices during the same period.
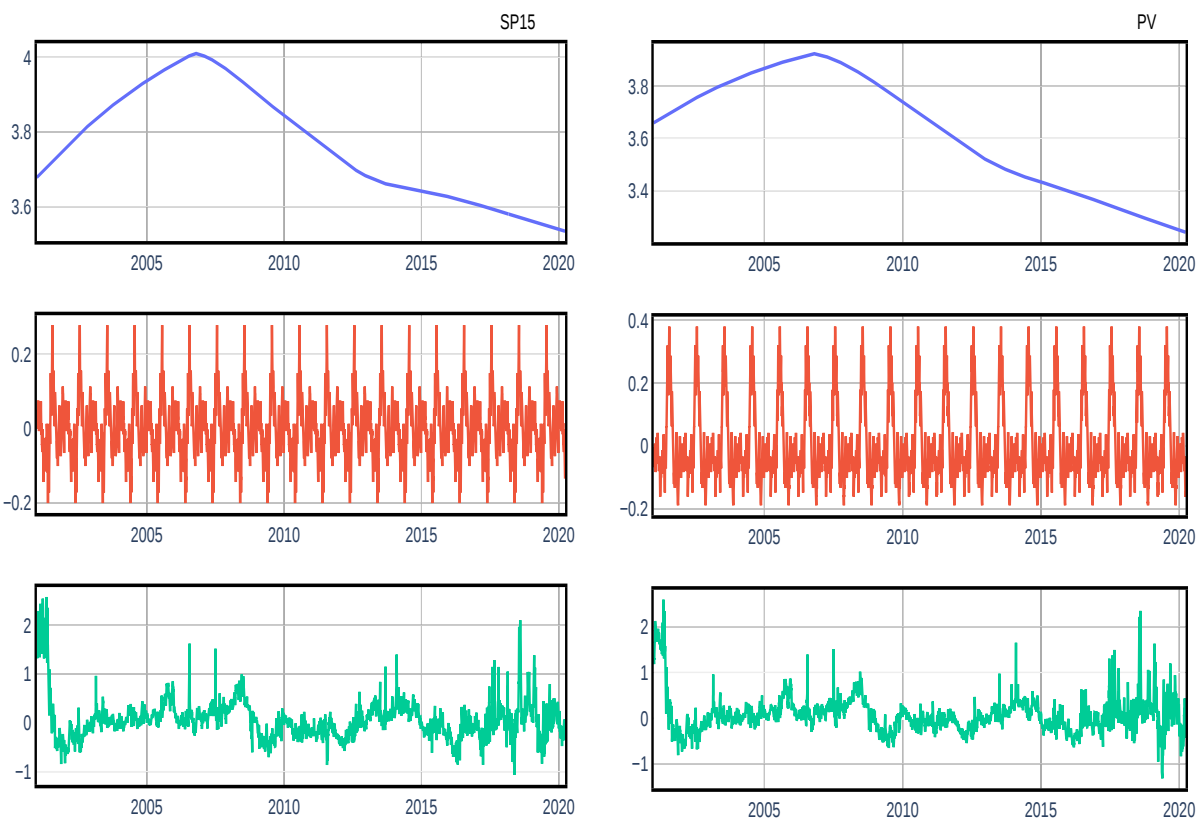

Figure 4. From top to bottom, the deterministic components: trend, seasonality and the stochastic component of the filled time series of daily electricity log-prices for the period 1 January 2001 to 24 March 2020 for SP15 and Palo Verde power markets. $x$-axis: calendar time; $y$-axis: singlecomponent log-price.

The anomaly detection problem can be now addressed. In the approach we propose, turbulent price movements, i.e., jumps and spikes, are identified as anomalies in the power price time dynamics. The purpose of this analysis is to identify and isolate anomalies in the stochastic component of the dynamics in order to decouple the stable motion from the jumpy and spiky behavior. To detect anomalies, we use the isolation forest (or iForest) algorithm which is an unsupervised learning algorithm for anomaly detection that works on the principle of isolating anomalies [34,35]. This method uses two main characteristics of anomalies: (i) they are the smaller part of the dataset and (ii) they have values that are very different from those that are considered normal points. Anomalies are "few and different," and these peculiarities allows us to isolate them with respect to normal data points. Being a technique that creates a data-induced random tree, called an isolation tree (or iTree), the data partitioning continues until the isolation of every instance has been obtained. As for their susceptibility to isolation, anomalies are more likely to be isolated closer to the root of the tree, while normal points stay more in depth. The iForest algorithm is characterized by high detection performances [34]. 
After detection, anomalies can be removed from the dynamics. At the end of this process, the additional gaps created by removing anomalies are newly filled by using the missForest imputation algorithm, thereby providing a new price time series describing the stable dynamics of electricity markets prices. We call this time series the "stable" time series. In this way, the stable motion can be decoupled from the turbulent motion in which jump and spikes are observed. Figure 6 shows both the stable dynamics (the red line) and the anomalous turbulent dynamics (the blue line) for the power markets under investigation. The analysis was performed in an unsupervised manner using the hyperparameters reported in Table 3. In Figure 7, we can see the detail for a 60 day time frame.
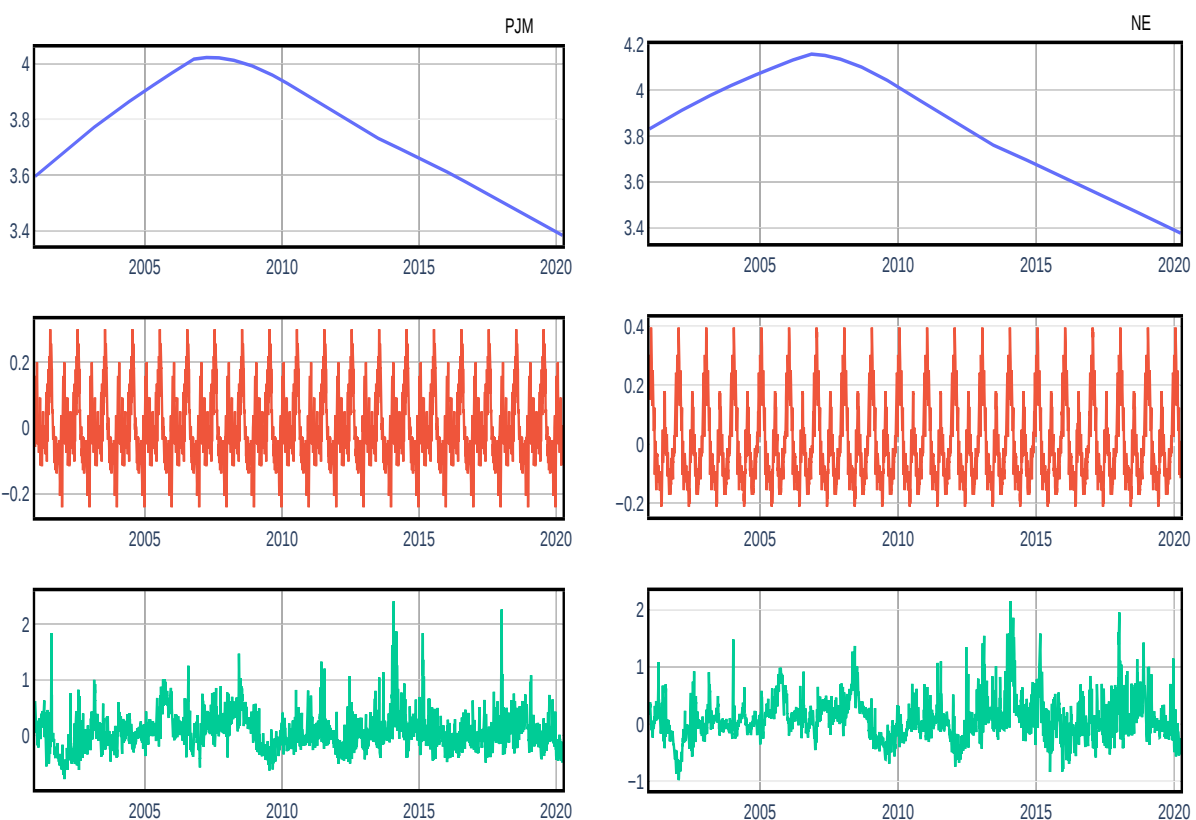

Figure 5. From top to bottom, the deterministic components: trend, seasonality and the stochastic component of the filled time series of daily electricity log-prices for the period 1 January 2001 to 24 March 2020 for PJM and Nepool power markets. $x$-axis: calendar time; $y$-axis: single-component log-price.

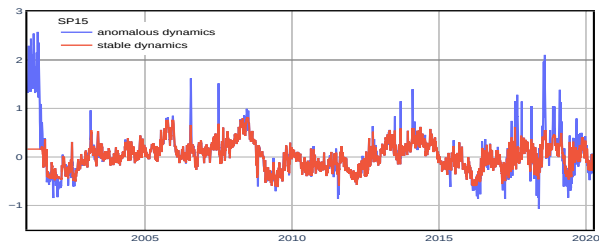

(a)

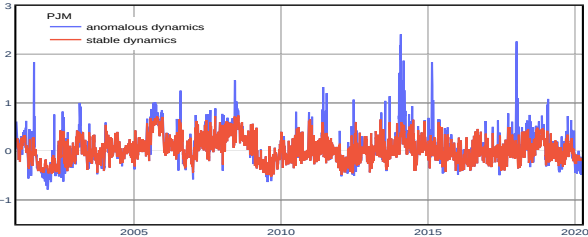

(c)

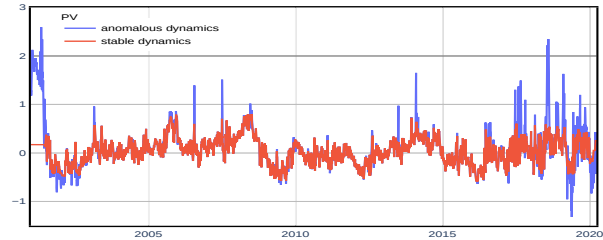

(b)

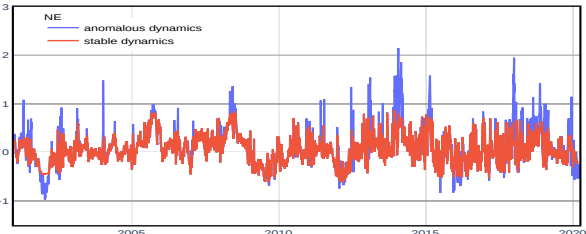

(d)

Figure 6. The decomposition of the stochastic dynamics of electricity market price: the stable dynamics (the red line) and the anomalous turbulent dynamics (the blue line). $x$-axis: calendar time; $y$-axis: stochastic component of log-price. (a) SP15; (b) Palo Verde; (c) PJM; (d) Nepool. 


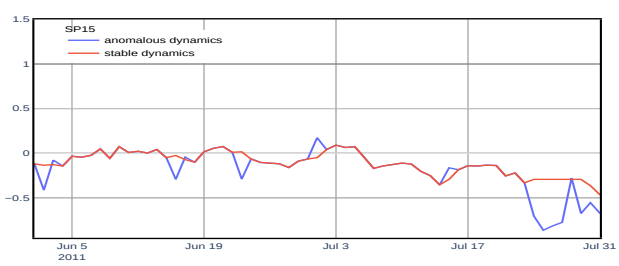

(a)

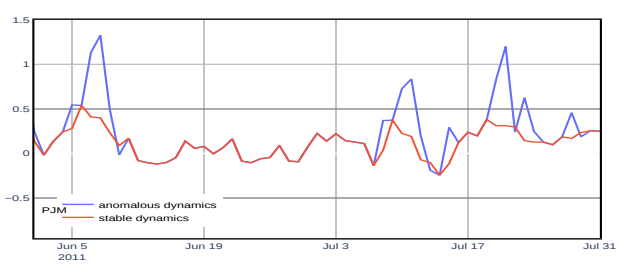

(c)

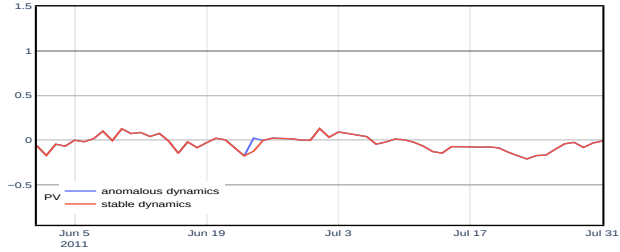

(b)

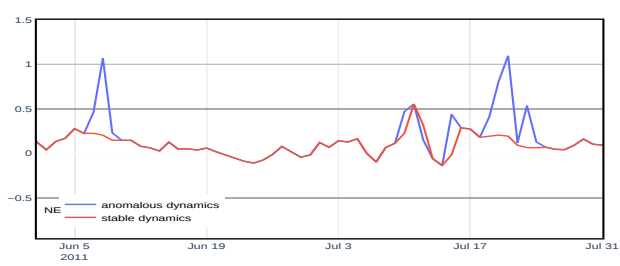

(d)

Figure 7. The decomposition of the stochastic dynamics of log-prices for the time interval June 2011 to July 2011: the stable dynamics (the red line) and the anomalous turbulent dynamics (the blue line). $x$-axis: calendar time; $y$-axis: stochastic component of log-price. (a) SP15; (b) Palo Verde; (c) PJM; (d) Nepool.

Table 3. iForest hyperparameter values.

\begin{tabular}{lc}
\hline iForest Hyperparameter & Value \\
\hline Number of iTrees & 1000 \\
Contamination value & Find automatically \\
Max samples & 256 \\
\hline
\end{tabular}

\section{Modeling Electricity Price Dynamics}

The reconstruction of the filled power price time series and the decoupling technique based on the machine learning algorithms are very useful for defining suitable stochastic models of the electricity price dynamics. Moreover, the decoupling between the stable motion and the turbulent motion allows us to introduce appropriate estimation techniques. In this section, we propose a mean-reverting jump-diffusion model of power prices and we discuss a two-step estimating technique of the model based on the information contained in both the stable time series and the turbulent dynamics.

\subsection{A Mean-Reverting Jump-Diffusion Model of Power Prices}

We focus on a mean-reverting jump-diffusion model in which the dynamics of $x(t)$ are described by the following stochastic differential equation:

$$
d x(t)=-\alpha x(t) d t+\sigma d w(t)+J d q(t)
$$

where $w(t)$ is a Wiener process and $q(t)$ is a Poisson process with constant intensity $\lambda$. In Equation (9) the random variable $J$, describing the jump amplitude, is assumed to be distributed as a normal random variable with mean $\mu$ and standard deviation $\sigma_{J}$, i.e., $J \sim N\left(\mu, \sigma_{J}^{2}\right)$. Moreover, we assumed that the Wiener process, the Poisson process and the jump amplitude, are mutually independent processes. Nevertheless, this analysis can be extended to account for jump amplitude with arbitrary probability distributions [53]. The decoupling technique discussed in this paper allows us to estimate the dynamic model using a two-step procedure that makes use of on the information contained in both the stable time series and the turbulent dynamics. We will show that the proposed model 
provides an interesting description of the power price dynamics observed in real markets, thereby offering a good compromise between mathematical tractability and the physical interpretation of the main stylized facts of power price dynamics. For this reason, it can be used for several financial applications ranging from the pricing of power derivatives and the hedging of financial risk [52], to the evaluation of long-term investments in power generating technologies [53].

\subsection{The Empirical Analysis}

Figure 8 reproduces the stochastic component of daily electricity prices, hereinafter, prices,

$$
p_{x}(t)=\exp (x(t))
$$

and the stochastic components of log-returns, hereinafter, log-returns,

$$
\Delta x(t)=x(t+\Delta t)-x(t),
$$

where $\Delta t$ is equal to one day, obtained from the original filled time series for the 15 -year period 1 January 2004 to 31 December 2018 for the four power markets under investigation.
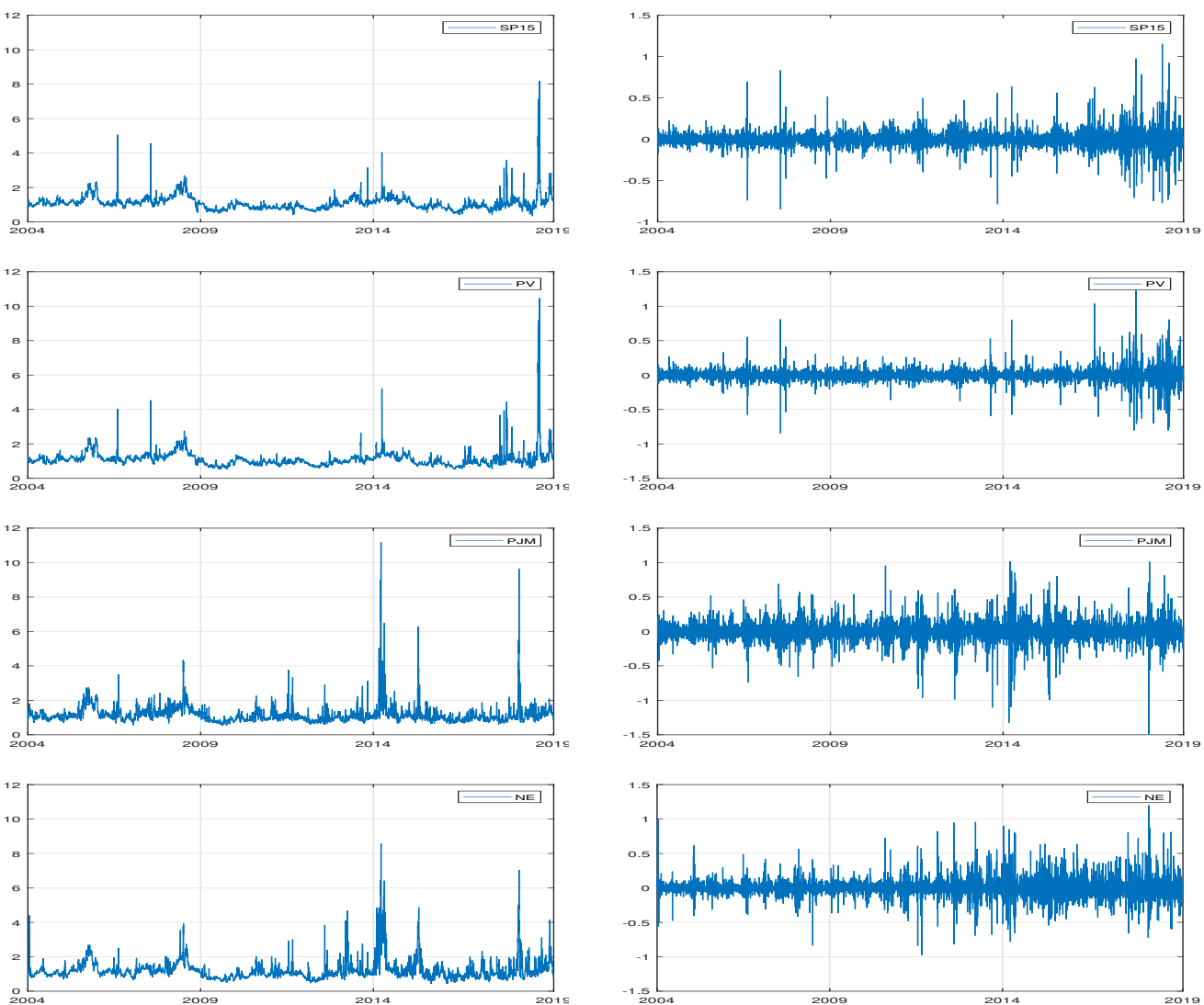

Figure 8. The time interval 1 January 2004 to 31 December 2018. Left panel: prices vs. calendar time. Right panel: log-returns vs. calendar time.

We note that in the power markets located in the same US region electricity prices show very similar patterns. Table 4 depicts the number of days with missing data in the 15-year period of 1 January 2004 to 31 December 2018. 
Table 4. Count of daily grid positions being empty (missing) or not (populated) for the period January 2004 to December 2018. Total grid positions: 5928.

\begin{tabular}{lcc}
\hline & Populated & Missing \\
\hline SP15 & 3628 & 1851 \\
PV & 3688 & 1791 \\
PJM & 3811 & 1668 \\
NE & 3555 & 1924 \\
\hline
\end{tabular}

\subsubsection{A Short-Term Empirical Analysis}

In this section we show an empirical analysis of the model in the 2-year time interval of 1 January 2017 to 31 December 2018. In all four markets, log-returns show large fluctuations with jumps and spikes, and non-normal, leptokurtic empirical distributions. The descriptive statistics of log-returns are displayed in Table 5.

Table 5. Descriptive statistics of log-returns (2017-2018).

\begin{tabular}{ccccc}
\hline & Mean & St. Dev. & Skewness & Kurtosis \\
\hline SP15 & 0.0001 & 0.1706 & 0.4441 & 11.5882 \\
PV & 0.0003 & 0.1768 & 0.3523 & 11.1401 \\
PJM & 0.0001 & 0.1585 & -0.4177 & 18.4634 \\
NE & -0.0004 & 0.1817 & 0.7832 & 8.7816 \\
\hline
\end{tabular}

We estimated the dynamics described by Equation (9) on market data by using a twostep procedure. In the first step, the parameters of the diffusion component of the model, i.e., $\alpha$ and $\sigma$, were estimated. In the second step the parameters of the jump component of the model, i.e., $\lambda, \mu$ and $\sigma_{J}$ were estimated.

In the first step, the following Euler discretization of the diffusion component of Equation (9) with time-step $\Delta t$ equal to one day was used, namely,

$$
\Delta x(t)=-\alpha x(t) \Delta t+\sigma \Delta w(t) .
$$

In order to account for the volatility due to the diffusion component of the model, without including the volatility of the jump component, the parameter $\sigma$ was determined by estimating Equation (12) on the stable time series (i.e., the red time series depicted in Figure 6) by maximum likelihood in the time interval 1 January 2017 to 31 December 2018. On the other hand, since the mean-reversion component must force back prices to fluctuate around the long-run mean after an anomalous price movement has occurred, the mean-reversion parameter, $\alpha$, was determined by estimating Equation (12) on the stochastic component of the filled original time series via maximum likelihood for the same time interval. Estimation results are depicted in Table 6.

Table 6. Estimation results (2017-2018): step one. Standard errors are between parentheses.

\begin{tabular}{ccccc}
\hline & $\alpha$ & & $\sigma$ & \\
\hline SP15 & 0.0838 & $(0.0097)$ & 0.0778 & $(0.0021)$ \\
PV & 0.0807 & $(0.0067)$ & 0.0883 & $(0.0023)$ \\
PJM & 0.1184 & $(0.0065)$ & 0.0885 & $(0.0024)$ \\
NE & 0.0852 & $(0.0064)$ & 0.1051 & $(0.0027)$ \\
\hline
\end{tabular}

In the second step of the estimation procedure we determined the values of the jump parameters. The approach we followed is based on the simulated moments method $[64,65]$ using Monte Carlo techniques $[65,66]$. For each triple of values $\left(\lambda, \mu, \sigma_{J}\right)$ belonging to a suitable three dimensional grid, a sample of one thousand random paths was generated 
from Equation (9) by using Monte Carlo techniques with the estimated parameters, $\alpha$ and $\sigma$, determined in the first step. Along each path, the first central moments, in particular the standard deviation, the skewness and the kurtosis, of log-returns were computed and averaged over the sample. We assumed that a triple $\left(\lambda, \mu, \sigma_{J}\right)$ offers a good fit if for each central moment, the difference between the sample average value and the observed value reported in Table 5 is less than one-fourth (25\%) of the sample standard deviation for that moment. Some good triples are reported in Table 7. Table 8 displays some statistical parameters of simulated paths. Such values are determined by averaging over one thousand randomly generated paths using the estimates obtained in the two-step procedure. The agreement with the descriptive statistics of log-returns shown in Table 5 is very interesting.

Table 7. Estimation results (2017-2018): step two.

\begin{tabular}{cccc}
\hline & $\mu$ & $\sigma_{J}$ & $\lambda$ \\
\hline SP15 & 0.04 & 0.37 & $15.8 \%$ \\
PV & 0.04 & 0.38 & $15.5 \%$ \\
PJM & -0.04 & 0.48 & $6.8 \%$ \\
NE & 0.09 & 0.35 & $15.8 \%$ \\
\hline
\end{tabular}

Table 8. Statistics of simulated path log-returns (2017-2018). Sample standard deviations are between parentheses.

\begin{tabular}{lcccc}
\hline & Mean & St. Dev. & Skewness & Kurtosis \\
\hline SP15 & -0.0001 & 0.1708 & 0.4609 & 11.7550 \\
& $(0.0005)$ & $(0.0113)$ & $(0.5264)$ & $(1.8673)$ \\
PV & 0.0000 & 0.1767 & 0.4036 & 11.1325 \\
& $(0.0006)$ & $(0.0113)$ & $(0.5067)$ & $(1.8445)$ \\
PJM & 0.0002 & 0.1582 & -0.4272 & 18.4418 \\
& $(0.0005)$ & $(0.0121)$ & $(0.9810)$ & $(4.3313)$ \\
NE & 0.0002 & 0.1813 & 0.7366 & 8.7964 \\
& $(0.0006)$ & $(0.0101)$ & $(0.3883)$ & $(1.3830)$ \\
\hline
\end{tabular}

We recall the importance, in option pricing and in risk hedging methodologies, of a given model being able to capture the first four central moments of empirical distributions of log-returns, not only the standard deviation. Skewness is particularly related to the asymmetry between upward versus downward moves; the kurtosis describes the tails of the distribution. These parameters are particularly relevant in the case of power prices in which extreme events may occur [52].

\subsubsection{A Long-Term Empirical Analysis}

Accurately modeling the electricity price dynamics using short-term models is also a crucial task for describing the electricity price dynamics on longer time horizons. In the presence of a mean-reverting component, in fact, the probability distributions of power prices tend to stationary long-run probability distributions [53]. In this way, the long-term behavior of power prices can be derived by the short-term dynamics. However, for a longrun empirical analysis, the amplitude of the estimation time interval must be increased in order to get more significant values [6]. To this end, we considered the 15-year period 1 January 2004 to 31 December 2018. The descriptive statistics of log-returns are displayed in Table 9. 
Table 9. Descriptive statistics of log-returns (2004-2018).

\begin{tabular}{ccccc}
\hline & Mean & St. Dev. & Skewness & Kurtosis \\
\hline SP15 & 0.0000 & 0.0960 & 0.4276 & 23.5549 \\
PV & 0.0000 & 0.0935 & 0.5753 & 28.2951 \\
PJM & 0.0000 & 0.1431 & -0.4011 & 14.7284 \\
NE & 0.0000 & 0.1360 & 0.4508 & 12.4794 \\
\hline
\end{tabular}

We used the same two-step procedure to estimate the jump-diffusion model described by Equation (9) over this fifteen-year time horizon too. Estimation results are depicted in Table 10 for the parameters of the diffusion component of the model, and in Table 11 for the parameters of the jump component of the model. Table 12 displays some statistical parameters computed from simulated log-return time series. Such values were determined by averaging over one thousand randomly generated paths using the estimates obtained in the two-step procedure. Additionally, in this case, the agreement with the descriptive statistics of observed log-returns shown in Table 9 is very interesting.

Table 10. Estimation results (2004-2018): step one. Standard errors are between parentheses.

\begin{tabular}{ccccc}
\hline & $\alpha$ & & $\sigma$ & \\
\hline SP15 & 0.0475 & $(0.0036)$ & 0.0613 & $(0.0007)$ \\
PV & 0.0479 & $(0.0011)$ & 0.0618 & $(0.0006)$ \\
PJM & 0.1008 & $(0.0027)$ & 0.0925 & $(0.0009)$ \\
NE & 0.0622 & $(0.0012)$ & 0.0886 & $(0.0009)$ \\
\hline
\end{tabular}

Table 11. Estimation results (2004-2018): step two.

\begin{tabular}{cccc}
\hline & $\mu$ & $\sigma_{J}$ & $\lambda$ \\
\hline SP15 & 0.03 & 0.34 & $4.5 \%$ \\
PV & 0.03 & 0.38 & $3.2 \%$ \\
PJM & -0.03 & 0.40 & $6.6 \%$ \\
NE & 0.04 & 0.34 & $8.5 \%$ \\
\hline
\end{tabular}

Table 12. Statistics of simulated path log-returns obtained using estimated parameters (2004-2018). Sample standard deviations are between parentheses.

\begin{tabular}{lcccc}
\hline & Mean & St. Dev. & Skewness & Kurtosis \\
\hline SP15 & 0.0000 & 0.0960 & 0.4560 & 23.5352 \\
& $(0.0001)$ & $(0.0034)$ & $(0.4844)$ & $(3.2481)$ \\
PV & 0.0000 & 0.0932 & 0.6194 & 28.5322 \\
& $(0.0001)$ & $(0.0034)$ & $(0.5851)$ & $(3.8200)$ \\
PJM & 0.0000 & 0.1425 & -0.4152 & 14.7151 \\
& $(0.0001)$ & $(0.0037)$ & $(0.3175)$ & $(1.4268)$ \\
NE & 0.0001 & 0.1356 & 0.4421 & $(12.4323$ \\
& $(0.0001)$ & $(0.0032)$ & $(0.2588)$ & \\
\hline
\end{tabular}

\section{Concluding Remarks}

In this paper we provided a general methodology to fill missing data in time series with irregular observation times and to detect anomalies in the dynamics. Our approach is based on machine learning ensemble techniques. In particular, the missForest imputation algorithm was used to fill in the gaps of the time series, and the isolation forest algorithm was used to detect anomalies in the time behavior. Moreover, the missForest algorithm was also used to fill the additional gaps originated by removing anomalies, in order to create a complete and clean time series describing the stable dynamics of power prices. 
The decoupling of the price dynamics between the stable motion and the turbulent motion allowed us to define a suitable mean-reverting jump-diffusion model of power prices and a two-step estimation procedure of the model parameters that uses the full information contained in both, the stable time series and the anomalous regions of the dynamics. The same two-step procedure was used to estimate both models, the short-term and the long-term.

The filling and decoupling technique proposed in this paper seems to be a powerful tool of analysis for investigating the features of the complex dynamics of power prices observed in real markets. It allows one to distinguish normal periods in which prices fluctuate around the long-run mean from turbulent movements of power prices characterized by jumps and spikes. Within this framework, the decoupling technique is a powerful tool for estimating jump-diffusion stochastic models of power prices in an accurate way. The obtained results show interesting agreement with empirical data.

Moreover, ensemble methods allowed us to put into evidence some similarities of the electricity price dynamics observed in different power markets. From this point of view, unsupervised machine learning techniques can be used to study the dynamics of the power markets prices as a whole, instead of taking them individually, thereby considering factors in common and similarities. We left those topics to future investigations.

Finally, let us remark that, although our analysis focused on power market prices, the proposed methodology is general and can be applied to very different contexts ranging from physical to social sciences.

Author Contributions: Conceptualization, C.M. and C.B.; methodology, C.M.; software, C.B.; validation, C.M. and C.B.; formal analysis, C.M.; investigation, C.M. and C.B.; data curation, C.B.; writing-original draft preparation, C.M. and C.B.; writing-review and editing, C.M. and C.B. All authors have read and agreed to the published version of the manuscript.

Funding: This research received no external funding.

Institutional Review Board Statement: Not applicable.

Informed Consent Statement: Not applicable.

Data Availability Statement: Data available in a publicly accessible repository.

Conflicts of Interest: The authors declare no conflict of interest.

\section{Appendix A. A Gap Filling Test for the missForest Algorithm}

In this section we provide a performance comparison between the missForest algorithm, used as gap filling technique for irregular time series, and a more traditional approach based on moving average techniques. To this end, we first randomly chose a time interval of August 2007 to December 2007. In this time interval, we created artificial gaps to be refilled using both the missForest algorithm and the moving average algorithm with a time window of 5, 10, 20 days, and we directly compared the filled data and true data during the same time periods. In this experiment, we artificially created a number of gaps equal to $5 \%, 10 \%$ and $15 \%$ of the number of market observations in the considered period. Then, for each couple of parameters, the experiment was repeated ten times to ensure that the artificial gaps had different patterns. Afterwards, to compare the two techniques we computed the MAPE (Mean Absolute Percentage Error), averaged for the ten rounds. The results are reported in Table A1. The outcomes show that in all cases the value of the $M A P E_{m i s s F o}$ is always consistently lower than the $M A P E_{m a}$, as we expected [55]. 
Table A1. The results of the gap filling test.

\begin{tabular}{|c|c|c|c|c|}
\hline & \multicolumn{2}{|c|}{ Parameters } & \multirow{2}{*}{$M A P E_{m a}$} & \multirow{2}{*}{ MAPE $E_{m i s s F o}$} \\
\hline & Fraction of Gaps & Time Window & & \\
\hline \multirow{9}{*}{ SP15 } & 5 & 5 & 10.05 & 6.21 \\
\hline & 5 & 10 & 8.38 & 5.05 \\
\hline & 5 & 20 & 12.84 & 5.25 \\
\hline & 10 & 5 & 9.62 & 6.07 \\
\hline & 10 & 10 & 10.64 & 5.54 \\
\hline & 10 & 20 & 11.61 & 4.57 \\
\hline & 15 & 5 & 7.92 & 4.7 \\
\hline & 15 & 10 & 11.21 & 6.78 \\
\hline & 15 & 20 & 11.93 & 5.36 \\
\hline \multirow{9}{*}{ PV } & 5 & 5 & 11.19 & 6.49 \\
\hline & 5 & 10 & 12.26 & 6.12 \\
\hline & 5 & 20 & 11.98 & 5.94 \\
\hline & 10 & 5 & 11.12 & 5.87 \\
\hline & 10 & 10 & 11.37 & 6.19 \\
\hline & 10 & 20 & 12.6 & 5.88 \\
\hline & 15 & 5 & 9.01 & 4.9 \\
\hline & 15 & 10 & 11.35 & 5.46 \\
\hline & 15 & 20 & 12.71 & 5.41 \\
\hline \multirow{9}{*}{ PJM } & 5 & 5 & 16.39 & 8.93 \\
\hline & 5 & 10 & 17.83 & 9.26 \\
\hline & 5 & 20 & 18.33 & 10.42 \\
\hline & 10 & 5 & 14.86 & 9.6 \\
\hline & 10 & 10 & 17.03 & 8.74 \\
\hline & 10 & 20 & 20.3 & 9.51 \\
\hline & 15 & 5 & 18.21 & 10.42 \\
\hline & 15 & 10 & 15.31 & 9.6 \\
\hline & 15 & 20 & 18.73 & 9.39 \\
\hline \multirow{9}{*}{$\mathrm{NE}$} & 5 & 5 & 10.38 & 7.74 \\
\hline & 5 & 10 & 10.11 & 5.32 \\
\hline & 5 & 20 & 14.3 & 6.29 \\
\hline & 10 & 5 & 12.94 & 8.13 \\
\hline & 10 & 10 & 12.83 & 7.43 \\
\hline & 10 & 20 & 14.02 & 7.57 \\
\hline & 15 & 5 & 10.21 & 7.38 \\
\hline & 15 & 10 & 11.03 & 6.79 \\
\hline & 15 & 20 & 14.85 & 7.78 \\
\hline
\end{tabular}

\section{References}

1. Robeson, S.M. Influence of sampling and interpolation on estimates of air temperature change. Clim. Res. 1994, 4, 119-126. [CrossRef]

2. Thiebaut, C.; Roques, S. Time-scale and time-frequency analyses of irregularly sampled astronomical time series. Eurasip J. Appl. Sig. Proc. 2005, 15, 2486-2499. [CrossRef]

3. Mateo, J.; Laguna, P. Improved heart rate variability signal analysis from the beat occurrence times according to the IPFM model. IEEE Trans. Biomed. Eng. 2000, 47, 985-996. [CrossRef]

4. Akansu, A.N.; Kulkarni, S.R.; Malioutov, D.M. Financial Signal Processing and Machine Learning; Wiley-IEEE Press: New York, NY, USA, 2016.

5. Owen, M. Practical Signal Processing; Cambridge University Press: Cambridge, UK, 2007.

6. Voit, J. The Statistical Mechanics of Financial Markets; Springer: Berlin/Heidelberg, Germany, 2005.

7. French, K.R. Stock returns and the week-end effect. J. Financ. Econ. 1980, 8, 55-69. [CrossRef] 
8. Mantegna, R.; Stanley, H.E. An Introduction to Econophysics-Correlations and Complexity in Finance; Cambridge University Press: Cambridge, UK, 2007.

9. Bahadori, M.T.; Liu, Y. Granger causality analysis in irregular time series. In Proceedings of the 12th SIAM International Conference on Data Mining, Anaheim, CA, USA, 26-28 April 2012.

10. Cuevas-Tello, J.C.; Tino, P.; Raychaudhury, S.; Yao, X.; Harva, M. Uncovering delayed patterns in noisy and irregularly sampled time series: An astronomy application. Pattern Recognit. 2009, 43, 1165-1179. [CrossRef]

11. Harteveld, W.K.; Mudde, R.F.; Van Den Akker, H.E.A. Estimation of turbulence power spectra for bubbly flows from laser Doppler anemometry signals. Chem. Eng. Sci. 2005, 60, 6160-6168. [CrossRef]

12. Kreindler, D.M.; Lumsden, C.J. The effects of the irregular sample and missing data in time series analysis. Nonlinear Dyn. Psychol. Life Sci. 2006, 10, 187-214.

13. Schulz, M.; Stattegger, K. Spectrum: Spectral analysis of unevenly spaced paleoclimatic time series. Comput. Geosci. 1997, 23, 929-945. [CrossRef]

14. Afrifa-Yamoah, E.; Mueller, U.A.; Taylor, S.M.; Fisher, A.J. Missing data imputation of high-resolution temporal climate time series data. Meteorol. Appl. 2020, 27, e1873. [CrossRef]

15. Ma, Q.; Li, S.; Shen, L.; Wang, J.; Wei, J.; Yu, Z.; Cottrell, G.W. End-to-end incomplete time series modeling from linear memory of latent variables. IEEE Trans. Cybern. 2019, 50, 4908-4920.

16. Suo, Q.; Yao, L.; Xun, G.; Sun, J.; Zhang, A. Recurrent imputation for multivariate time series with missing values. In Proceedings of the IEEE International Conference on Healthcare Informatics, Xi'an, China, 10-13 June 2019.

17. Bertsimas, D.; Pawlowski, C.; Zhuo, Y. From predictive methods to missing data imputation: An optimization approach. JMLR 2018, 18, 1-39.

18. Che, Z.; Purushotham, S.; Cho, K.; Sontag, D.; Liu, Y. Recurrent neural networks for multivariate time series with missing values. Sci. Rep. 2018, 8, 2045-2322. [CrossRef] [PubMed]

19. Broersen, P.M.T. Spectral analysis of irregularly sampled data with time series models. Open Signal Process. J. 2008, 1, 7-14. [CrossRef]

20. Mahmoudvand, R.; Rodrigues, P.C. Missing value imputation in time series using Singular Spectrum Analysis. Int. J. Energy Stat. 2016, 4, 1650005. [CrossRef]

21. Foster, G. Wavelets for period analysis of unevenly sampled time series. Astron. J. 1996, 112, 1709-1729. [CrossRef]

22. Mondal, D.; Percival, D.B. Wavelet variance analysis for gappy time series. Ann. Inst. Stat. Math. 2008, 62, 943-966. [CrossRef]

23. Sweldens, W. The lifting scheme: A construction of second generation wavelets. SIAM J. Math. Anal. 1998, 29, 511-546. [CrossRef]

24. Wilson, R.E.; Eckley, I.A.; Matthew, A.N.; Park, T. A wavelet-based approach for imputation in nonstationary multivariate time series. Stat. Comput. 2021, 31, 1-18. [CrossRef]

25. Mikalsena, K.Ø.; Bianchi, F.M.; Soguero-Ruizb, C.; Jenssenb, R. Time series cluster kernel for learning similarities between multivariate time series with missing data. Pattern Recognit. 2018, 76, 569-581. [CrossRef]

26. Rehfeld, K.; Marwan, N.; Heitzig, J.; Kurths, J. Comparison of correlation analysis techniques for irregularly sampled time series. Nonlinear Process. Geophys. 2011, 18, 389-404. [CrossRef]

27. Stekhoven, D.J.; Bühlmann, P. MissForest-Non-parametric missing value imputation for mixed-type data. Bioinformatics 2012, 28, 112-118. [CrossRef]

28. Hawkins, D.M. Identification of Outliers; Springer: Berlin/Heidelberg, Germany, 1980.

29. General Accounting Office. Additional Actions Would Help Ensure that FERC's Oversight and Enforcement Capability Is Comprehensive and Systematic; GAO-03-845; General Accounting Office: Washington, DC, USA, 2003.

30. Chen, H. Power Grid Operation in a Market Environment: Economic Efficiency and Risk Mitigation; Wiley-IEEE Press: New York, NY, USA, 2017.

31. Eydeland, A.; Wolyniec, K. Energy and Power Risk Management; Wiley: Chichester, UK, 2003.

32. Weron, R. Modeling and Forecasting Electricity Loads and Prices: A Statistical Approach; Wiley: Chichester, UK, 2013.

33. Geman, H.; Roncoroni, A. Understanding the fine structure of electricity prices. J. Bus. 2006, 79, 1225-1261. [CrossRef]

34. Liu, F.T.; Ting, K.M.; Zhou, Z.H. Isolation Forest. In Proceedings of the Eighth IEEE International Conference on Data Mining, Pisa, Italy, 15-19 December 2008; doi: 10.1109/ICDM.2008.17. [CrossRef]

35. Ezziyyani, M. Advanced Intelligent Systems for Sustainable Development; Springer: Berlin/Heidelberg, Germany, 2020.

36. Emmott, A.; Das, S.; Dietterich, T.; Fern, A.; Wong, W. A meta-analysis of the anomaly detection problem. arXiv 2015, arXiv:1503.01158.

37. Aggarwal, C.C.; Saket, S. Outlier Ensembles: An Introduction; Springer International Publishing AG: Dordrecht, The Netherlands, 2017; Chapter 6, ISBN 978-3-319-54765-7.

38. Ting, K.M.; Aryal, S.; Washio, T. Which outlier detector should I use? In Proceedings of the 2018 IEEE International Conference on Data Mining (ICDM), Singapore, 17-20 November 2018.

39. Lucia, J.; Schwartz, E.S. Electricity prices and power derivatives: Evidence from the Nordic power exchange. Rev. Deriv. Res. 2002, 5, 5-50. [CrossRef]

40. Cartea, A.; Figuera, M. Pricing in electricity markets: A mean reverting jump diffusion model with seasonality. Appl. Math. Financ. 2005, 12, 313-335. [CrossRef] 
41. Kegnenlezom, M.; Takam Soh, P.T.; Mbele Bidima, M.L.D.; Emvudu Wono, Y. A jump-diffusion model for pricing electricity under price-cap regulation. Math. Sci. 2019, 13, 395-405. [CrossRef]

42. Hamilton, J.D. A new approach to the economic analysis of nonstationary time series and the business cycle. Econometrica 1989, 57, 357-384. [CrossRef]

43. Huisman, R.; Mahieu, R. Regime jumps in electricity prices. Energy Econ. 2003, 25, 423-434. [CrossRef]

44. Weron, R.; Bierbrauer, M.; Trück, S. Modeling electricity prices: Jump-diffusion and regime switching. Physica A 2004, 336, 39-48. [CrossRef]

45. Mari, C. Regime-switching characterization of electricity prices dynamics. Physica A 2006, 371, 552-564. [CrossRef]

46. Meyer-Brandis, T.; Tankov, P. Multi-factor Jjump-diffusion models of electricity prices. Int. J. Theor. Appl. Financ. 2008, 11, 503-528. [CrossRef]

47. Akouemo, H.N.; Povinelli, R.J. Data improving in time series using ARX and ANN models. IEEE Trans. Power Syst. 2017, 32, 3352-3359. [CrossRef]

48. Pereira, J.; Silveira, M. Unsupervised anomaly detection in energy time series data using variational recurrent autoencoders with attention. In Proceedings of the 17th IEEE International Conference on Machine Learning and Applications (ICMLA), Orlando, FL, USA, 17-20 December 2018; pp. 1275-1282.

49. Zor, K.; Çelik, Ö.; Timur, O.; Yildirim, H.B.; Teke, A. Simple approaches to missing data for energy forecasting applications. In Proceedings of the 16th International Conference on Clean Energy, Gazimağusa, Cyprus, 9-11 May 2018.

50. Zhang, J.; Tan, Z.; Yang, S. Day-ahead electricity price forecasting by a new hybrid method. Comput. Ind. Eng. 2012, 63, 695-701. [CrossRef]

51. Weron, R. Electricity price forecasting: A review of the state-of-the-art with a look into the future. Int. J. Forecast. 2014, 30, 1030-1081. [CrossRef]

52. Geman, H. Commodities and Commodity Derivatives; Wiley: Chichester, UK, 2005.

53. Mari, C. Short-term movements of electricity prices and long-term investments in power generating technologies. Energy Syst. 2021. [CrossRef]

54. Breiman, L. Random forests. Mach. Learn. 2001, 45, 5-32. [CrossRef]

55. Misztal, M. Some Remarks on the Data Imputation Using 'missForest' Method. Acta Univ. Lodz. Folia Oecon. 2013. Available online: http:/ / polona.pl/item/45700373 (accessed on 8 April 2021).

56. Little, R.J.A.; Rubin, D.B. Statistical Analysis with Missing Data; John Wiley \& Sons: New York, NY, USA, 1986.

57. Beguería, S.; Tomas-Burguera, M.; Serrano-Notivoli, S.; Peña-Angulo, D.; Vicente-Serrano, S.M.; González-Hidalgo, J.-C. Gap Filling of Monthly Temperature Data and Its Effect on Climatic Variability and Trends. J. Clim. 2019, 32, 7797-7821. [CrossRef]

58. Shah, A.D.; Bartlett, J.W.; Carpenter, J.; Nicholas, O.; Hemingway, H. Comparison of random forest and parametric imputation models for imputing missing data using MICE: A CALIBER study. Am. J. Epidemiol. 2014, 179, 764-774. [CrossRef]

59. Chenguang, F.; Chen, W. Time Series Data Imputation: A Survey on Deep Learning Approaches. arXiv 2020, arXiv:2011.11347.

60. Waljee, A.K.; Mukherjee, A.; Singal, A.G.; Zhang, Y.; Warren, J.; Balis, U.; Marrero, J.; Zhu, J.; Higgins, P. Comparison of imputation methods for missing laboratory data in medicine. BJM Open 2013, 3, e002847. [CrossRef] [PubMed]

61. Bauer, J.; Angelini, O.; Denev, A. Imputation of Multivariate Time Series Data-Performance Benchmarks for Multiple Imputation and Spectral Techniques. 2017. Available online: https://ssrn.com/abstract=2996611 (accessed on 30 November 2020).

62. Cleveland, R.B.; Cleveland, W.S.; McRae, J.E.; Terpenning, I.J. STL: A seasonal-trend decomposition procedure based on LOESS. J. Off. Stat. 1990, 6, 3-33.

63. Dagum, E.B.; Bianconcini, S. Seasonal Adjustment Methods and Real Time Trend-Cycle Estimation; Springer: Berlin/Heidelberg, Germany, 2016.

64. Duffie, D.; Singleton, K. Simulated moments estimation of Markov models of asset prices. Econometrica 1993, 61, 929-952. [CrossRef]

65. McFadden, M. A method of simulated moments for estimation of discrete response models without numerical integration. Econometrica 1989, 57, 995-1026. [CrossRef]

66. Gelman, A. Method of moments using Monte Carlo simulation. J. Comput. Graph. Stat. 1995, 4, 36-54. 